\title{
Using electron scattering to constrain the axial-vector form factor
}

\section{R. González-Jiménez*}

Departamento de Física Atómica, Molecular y Nuclear, Universidad de Sevilla, 41080 Sevilla, Spain

E-mail: raugj@us.es

\section{J. A. Caballero}

Departamento de Física Atómica, Molecular y Nuclear, Universidad de Sevilla, 41080 Sevilla, Spain

\section{T. W. Donnelly}

Center for Theoretical Physics, Laboratory for Nuclear Science and Department of Physics,

Massachusetts Institute of Technology, Cambridge, Massachusetts 02139, USA

\begin{abstract}
We present an analysis of elastic and quasielastic parity-violating (PV) electron scattering processes. These reactions can help to constrain the weak neutral current form factors of the nucleon that play an essential role in the description of neutrino cross sections at intermediate energies. We show that combining information from the analysis of elastic and quasielastic reactions the current knowledge on the strange and axial-vector form factors can be significantly improved.
\end{abstract}

16th International Workshop on Neutrino Factories and Future Neutrino Beam Facilities - NUFACT2014, 25 -30 August, 2014

University of Glasgow, United Kingdom

${ }^{*}$ Speaker. 


\section{Introduction}

Motivated by the discovery of neutrino oscillations, recent years have been marked by a huge activity and new initiatives in both experimental and theoretical neutrino physics. Most of the neutrino scattering experiments that have been proposed or recently carried out involve nuclear targets. Therefore, a good understanding of the neutrino-nucleus cross sections is essential in order to reduce the uncertainties in the determination of the oscillation parameters. Many of these experiments (see [1]), MiniBooNE, SciBooNE, Minerva, NOMAD, K2K, T2K, have been designed to work at the intermediate energy regime (from hundreds of $\mathrm{MeV}$ to a few $\mathrm{GeV}$ ) where the quasielastic $(\mathrm{QE})$ process is one of the dominant channels in the reaction mechanism. In this energy range, nucleon form factors play a fundamental role in the description of the cross section. In this work we aim to show that parity-violating electron scattering can be used to study the form factors that enter in the weak neutral current of the nucleon.

The use of electrons as projectiles in comparison to neutrinos has important advantages: i) electrons are easily produced, accelerated and detected, and ii) it is possible to produce monochromatic beams. In particular, the latter (monochromatic beams) allows one to have better control of the kinematics, since it is easier to estimate which specific channels are involved in the observed cross section (quasielastic, resonances, deep inelastic scattering, etc.).

In parity-violating electron scattering experiments a longitudinally polarized electron is scattered from a nucleon (proton) or a nucleus, the electron being detected in the final state. The Feynman diagrams describing the scattering process (in Born approximation) are shown in Fig. 1. Although the electromagnetic (EM) interaction, mediated by the exchange of a virtual photon (diagram (a)), is dominant, the electron also interacts with the target through the weak neutral current (WNC) interaction, mediated by the exchange of a virtual $Z^{0}$ boson (diagram (b)). Therefore, the cross section $(\sigma)$ consists of the sum of three terms; the pure EM contribution, an interference term between the EM and WNC currents and a purely WNC contribution:

$$
\sigma \propto\left|\mathscr{M}_{\gamma}+\mathscr{M}_{Z}\right|^{2}=\left|\mathscr{M}_{\gamma}\right|^{2}+2 \mathscr{R} e\left(\mathscr{M}_{\gamma}^{*} \mathscr{M}_{Z}\right)+\left|\mathscr{M}_{Z}\right|^{2}
$$

where $\mathscr{M}_{\gamma}=j_{\gamma}^{\mu} J_{\mu}^{\gamma}$ with $j_{\gamma}^{\mu}\left(J_{\mu}^{\gamma}\right)$ the EM leptonic (hadronic) current. Similarly, $\mathscr{M}_{Z}=j_{Z}^{\mu} J_{\mu}^{Z}$, with $j_{Z}^{\mu}\left(J_{\mu}^{Z}\right)$ the WNC leptonic (hadronic) current.

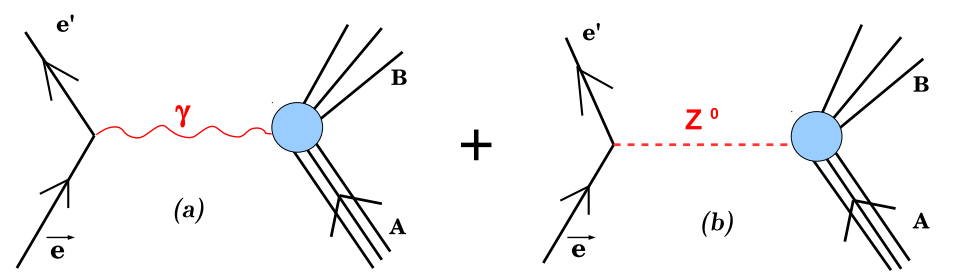

Figure 1: First-order Feynman diagrams for PV electron scattering: (a) one photon exchanged, EM interaction, (b) one $Z^{0}$-boson exchanged, WNC interaction.

The parity-violating asymmetry $\left(\mathscr{A}^{P V}\right)$ is defined as

$$
\mathscr{A}^{P V}=\frac{\sigma^{+}-\sigma^{-}}{\sigma^{+}+\sigma^{-}}=\frac{\sigma^{P V}}{\sigma^{P C}},
$$


where $\sigma^{+/-}$represents the cross section with positive/negative helicity of the incident electron. On the one hand, the denominator in the asymmetry is dominated by the EM contribution, $\sigma^{P C} \propto$ $\left|\mathscr{M}_{\gamma}\right|^{2}$, that is, a parity conserving (PC) cross section. On the other hand, the first-order contribution in the numerator is the EM-WNC interference term, with the purely WNC contribution being several orders of magnitude smaller. Thus, $\sigma^{P V} \propto 2 \mathscr{R} e\left(\mathscr{M}_{\gamma}^{*} \mathscr{M}_{Z}\right)$, i.e., a parity-violating (PV) cross section. Notice that the PV asymmetry is different from zero due exclusively to the presence of the weak interaction. For this reason, the PV asymmetry can be used to study the different ingredients that enter in the weak neutral current. In particular, in this work we focus on the analysis of the WNC form factors of the nucleon, paying special attention to the axial-vector one.

We analyze two different processes, PV elastic electron-nucleon scattering (PVE), section 2, and PV quasielastic electron-nucleus scattering (PVQE), section 3. In the former, we have performed a statistical analysis of the full set of PVE asymmetry data (elastic electron scattering off proton $[2,3,4,5,6,7,8,9,10,11,12]$ and $\left.{ }^{4} \mathrm{He}[5,13]\right)$ providing estimates on the WNC form factors, in particular, on the electric (E) and magnetic (M) strange form factors of the nucleon $\left(G_{E, M}^{(s)}\right)$ and on the axial-vector one $\left(G_{A}^{e p}\right)$. In section 3 we present a brief discussion on the PVQE asymmetry and show how this observable could provide information on the WNC nucleon form factors that complements what is obtained from the elastic reaction. In particular, it is shown that this observable could help to constrain the isovector contribution in the axial-vector form factor [14].

\section{Parity-violating elastic electron-proton scattering}

After some algebra (see [15] for details), the parity-violating elastic electron-proton asymmetry $\left(\mathscr{A}_{e p}^{P V}\right)$ can be written in the form:

$$
\mathscr{A}_{e p}^{P V}=-\frac{\mathscr{A}_{0}}{2}\left[\xi_{V}^{p}+\frac{G^{p n}}{G^{p p}} \xi_{V}^{n}+\frac{\xi_{V}^{(0)} \varepsilon G_{E}^{p}}{G^{p p}} G_{E}^{(s)}+\frac{\xi_{V}^{(0)} \tau G_{M}^{p}}{G^{p p}} G_{M}^{(s)}-\frac{\left(1-4 \sin ^{2} \theta_{W}\right) \delta^{\prime} G_{M}^{p}}{G^{p p}} G_{A}^{e p}\right],
$$

where $\mathscr{A}_{0}$ is a function of the four-momentum transferred, $Q^{2}$, that determines the scale of the process. $\delta^{\prime}=\sqrt{\left(1-\varepsilon^{2}\right) \tau(1+\tau)}$, being $\tau$ and $\varepsilon$ kinematic factors (see ref. [15] for explicit expressions). $\theta_{W}$ is the weak mixing angle and the quantities $\xi_{V}$ are the WNC effective vector couplings. Finally, $G_{E, M}^{p, n}$ are the EM form factors of the nucleon and the functions $G^{p p}=\varepsilon\left(G_{E}^{p}\right)^{2}+\tau\left(G_{M}^{p}\right)^{2}$ and $G^{p n}=\varepsilon G_{E}^{p} G_{E}^{n}+\tau G_{M}^{p} G_{M}^{n}$ have been introduced.

The axial-vector form factor can be decomposed in terms of a dominant isovector contribution $\left(G_{A}^{(3)}\right)$ and two (octet, $G_{A}^{(8)}$, and strangeness, $G_{A}^{(s)}$ ) isoscalar contributions:

$$
G_{A}^{e p}=\xi_{A}^{T=)} G_{A}^{(3)}+\xi_{A}^{T=0} G_{A}^{(8)}+\xi_{A}^{(s)} G_{A}^{(s)} .
$$

Here the terms $\xi_{A}$ represent the WNC effective axial-vector couplings.

In the case of elastic electron- ${ }^{4} \mathrm{He}$ scattering the PV asymmetry can be written as (see [16]):

$$
\mathscr{A}_{e H e}^{P V}=-\frac{\mathscr{A}_{0}}{2}\left[\xi_{V}^{p}+\xi_{V}^{n}+\frac{2 \xi_{V}^{(0)}}{G_{E}^{p}+G_{E}^{n}} G_{E}^{(s)}\right] .
$$

As observed, $\mathscr{A}_{e p}^{P V}$ (eq. (2.1)) depends on the strange and axial-vector form factors, while $\mathscr{A}_{e H e}^{P V}$ (eq. (2.3)) depends on the electric strangeness. Consequently, a statistical analysis of the available 
experimental data on these observables provides information on the WNC nucleon structure. As already mentioned in the introduction, this is of great relevance for the analysis of neutral-current (NC) neutrino scattering reactions at intermediate energies.

Some considerations are needed regarding the axial-vector form factor and the WNC effective couplings. Corrections to the cross section from higher-order contributions, namely, radiative corrections (RC), are usually included in the WNC effective couplings $(\xi)$ by modifying their tree-level values (see [16]). However, a theoretical evaluation of these RC is not yet free from ambiguities. In fact, the contribution of RC is one of the main sources of uncertainties in the analysis of the PVE asymmetry. In particular, contrary to neutrino scattering reactions where only the weak couplings are involved, in PV electron scattering, RC may play a very significant role in the description of the nucleon axial current and, consequently, in the axial-vector form factor.

At tree-level the value of the axial-vector form factor is ${ }^{1} G_{A}^{\text {tree }}\left(Q^{2}\right)=-1.19 G^{A}\left(Q^{2}\right)$, where $G^{A}\left(Q^{2}\right)$ is a function of the four-momentum transferred. According to the study of RC presented in ref. [19], the previous result should be modified to $G_{A}^{e p}\left(Q^{2}\right)=(-1.04 \pm 0.44) G^{A}\left(Q^{2}\right)$, its uncertainty being directly linked to the RC uncertainties. Thus, summarizing, RC may modify the tree-level value of $G_{A}^{e p}\left(Q^{2}\right)$ by more than $12 \%$, introducing also an additional uncertainty of the order of $50 \%$.

In the present work we revisit the results from the statistical analysis of PVE asymmetry data presented in [20]. The EM form factors of the nucleon are assumed to be well under control, being described by the GKex prescription [21, 22, 23] (see [15] for a detailed discussion on this topic). Moreover, in order to include in our analysis data corresponding to a wide range of $Q^{2}$ values $\left(0.02<\left|Q^{2}\right|<1(\mathrm{GeV} / \mathrm{c})^{2}\right)$ the following $Q^{2}$-functional dependence of the strange and axial-vector form factors were used:

$$
G_{E}^{(s)}\left(Q^{2}\right)=\rho_{s} \tau G_{D}^{V}\left(Q^{2}\right), G_{M}^{(s)}\left(Q^{2}\right)=\mu_{s} G_{D}^{V}\left(Q^{2}\right), G_{A}^{e p}=G_{A}^{e p}(0) G_{D}^{A}\left(Q^{2}\right)
$$

where $G_{D}^{V}\left(Q^{2}\right)=\left(1+\left|Q^{2}\right| / M_{V}^{2}\right)^{-2}$ and $G_{D}^{A}\left(Q^{2}\right)=\left(1+\left|Q^{2}\right| / M_{A}^{2}\right)^{-2}$, with $M_{V}=0.84 \mathrm{GeV} / \mathrm{c}^{2}$ and $M_{A}=1.03 \mathrm{GeV} / \mathrm{c}^{2}$.

The analysis consists in fitting simultaneously the electric $\left(\rho_{s}\right)$ and magnetic $\left(\mu_{s}\right)$ strangeness parameters, the WNC effective couplings of the proton $\left(\xi_{V}^{p}\right)$ and neutron $\left(\xi_{V}^{n}\right)$ and the value of the axial-vector form factor at zero four-momentum transferred $\left(G_{A}^{e p} \equiv G_{A}^{e p}(0)\right)$. The linear dependence of the PV asymmetries (see eqs. (2.1) and (2.3)) on the free parameters: $\rho_{s}, \mu_{s}, G_{A}^{e p}, \xi_{V}^{p}$ and $\xi_{V}^{n}$, simplifies importantly the problem since it is possible to perform an analytical $\chi^{2}$ fit (see [24]). Finally, notice that using $\xi_{V}^{p, n}$ and $G_{A}^{e p}$ as free parameters avoids introducing systematical errors linked to RC. Moreover, it also allows us to estimate the potential contributions from RC in the axial and vector sectors of the current by comparing the results from the fit to their tree-level values.

In Fig. 2 we present the comparison of the theoretical PV asymmetry and the experimental data. Each panel corresponds to a different scattering angle and the asymmetry is represented as a function of the four momentum transferred. In panel (f) the PV electron- ${ }^{4} \mathrm{He}$ asymmetry is shown while the rest of panels correspond to the PV electron-proton asymmetry. The grey band represents

${ }^{1}$ The values $G_{A}^{(3)}(0) \equiv g_{A}=1.27$ and $G_{A}^{(s)}(0) \equiv g_{A}^{(s)}=-0.08[17,18]$ has been assumed. 


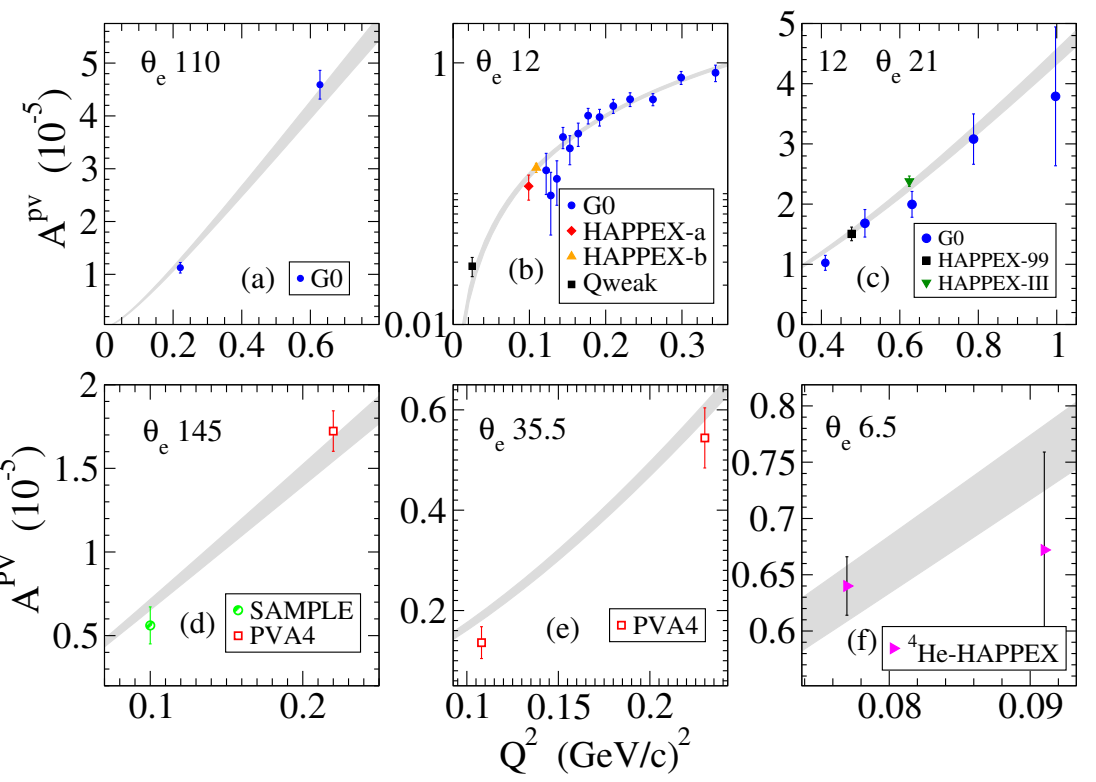

Figure 2: Full set of PV asymmetry data for elastic electron scattering compared with the prediction from the $\chi^{2}$-fit (grey band). The width of the band represents the theoretical uncertainty (1- $\sigma$ error). The reduced- $\chi^{2}$ value is 1.30 .

the $1 \sigma$ error from the fit. As observed, the agreement with data is rather good, particularly, at forward scattering angles (panels (b) and (c) $)^{2}$.
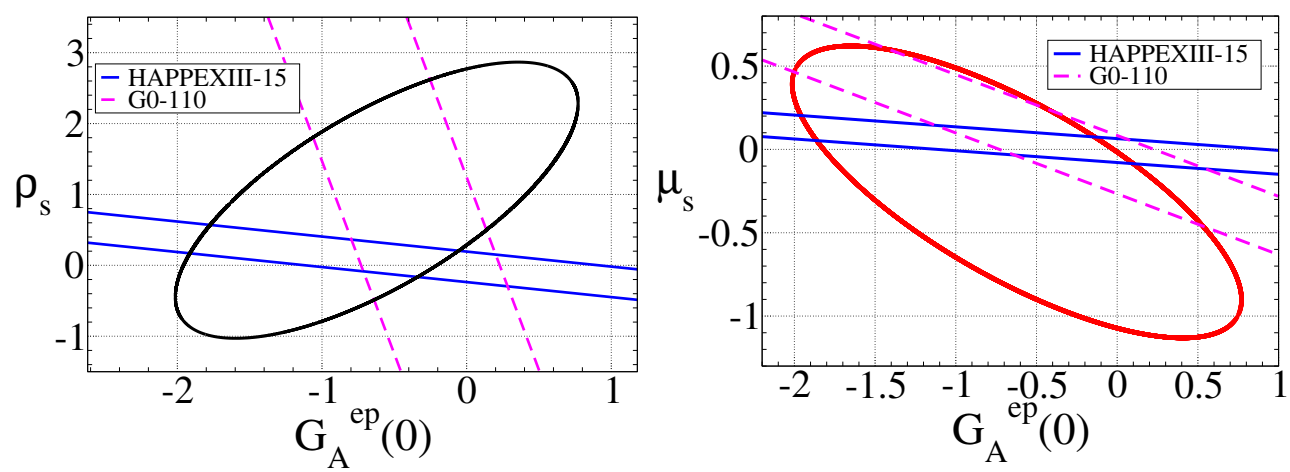

Figure 3: 95\% confident level contours in the $\left[\rho_{s}-G_{A}^{e p}\right]$ (left) and $\left[\mu_{s}-G_{A}^{e p}\right]$ (right) planes. The correlation coefficient for the couple $\rho_{s} \leftrightarrow G_{A}^{e p}$ is 0.711 while for $\mu_{s} \leftrightarrow G_{A}^{e p}$ is -0.749 . The straight lines represent the constraints from two experimental data at forward (HAPPEXIII-15 $\left.{ }^{\circ}[6]\right)$ and backward (G0-110 [11]) scattering angles.

In Fig. 3 we represent the $95 \%$ confident level ellipses for the parameters $\rho_{s}, \mu_{s}$ and $G_{A}^{e p}$. The central values (point of maximum likelihood) and correlation coefficients for the full set of free parameters as well as other confident level ellipses have been presented in refs. [20, 25]. Here we only discuss the results concerning the axial-vector form factor for which one gets $G_{A}^{e p}=-0.62 \pm 0.41$. Additionally, we have represented the constraints from two data, HAPPEXIII and G0- $110^{\circ}$, as examples of the two limit situations: forward and backward scattering. In the $\rho_{s}-G_{A}^{e p}$ plane (left panel) the band corresponding to the backward data is almost vertical, that is, backward data

\footnotetext{
${ }^{2}$ Notice that the high precision forward data, HAPPEX-III, HAPPEX-a, HAPPEX-b, dominate the fit.
} 
provide essential information on the axial-vector form factor but not on $\rho_{s}$. The opposite occurs at forward scattering, where data (horizontal band) basically constrain the electric strangeness parameter. In the $\mu_{s}-G_{A}^{e p}$ plane (right panel) the situation is a somewhat different. Forward scattering data essentially constrain $\mu_{s}$ but not $G_{A}^{e p}$ while at backward kinematics results are sensitive to both $\mu_{s}$ and $G_{A}^{e p}$.

\section{Parity-violating quasielastic electron-nucleus scattering}

In this section we study the inclusive parity-violating quasielastic electron-nucleus scattering process, $A\left(\vec{e}, e^{\prime}\right) B$. Within the $\mathrm{QE}$ regime one considers that the longitudinally polarized electron interacts with only one nucleon in the target nucleus, with the struck nucleon being ejected from the nucleus. Inclusive refers to the fact that the only detected particle is the final electron. In any other situation, for instance, in the exclusive case $^{3}$, the pure EM responses also contribute to the numerator of the PV asymmetry (1.2) making this observable useless to study the weak neutral current (see [26]).

In the left panel of Fig. 4 we present the PVQE asymmetry computed with different nuclear models based on the impulse approximation:

- Relativistic Fermi gas (RFG) [27]. The initial and final states of the struck nucleon are described as free-Dirac spinors.

- Relativistic plane-wave impulse approximation (RPWIA) [14, 26]. The bound nucleon wave function is a solution of the Relativistic Mean Field (RMF) Dirac equation while the scattered nucleon is a Dirac plane wave.

- Relativistic mean field with final-state interactions (RMF-FSI) [14]. The bound and scattered nucleon wave functions are solutions of the same RMF Dirac equation.

We see that the PVQE asymmetry is quite insensitive to the final state interactions (RPWIA vs RMF-FSI) and also to the description of the initial state of the nucleon (RPWIA vs RFG). In particular, in the region around the center of the QE-peak, $\omega \approx 500 \mathrm{MeV}$, the results of the three models deviate less than $\sim 7 \%$.

We have also studied the sensitivity of the PVQE asymmetry to nucleonic effects [14]. In particular, at backward scattering angles the PVQE asymmetry shows special sensitivity to the description of the magnetic and axial-vector form factors. In the right panel of Fig. 4, the effect of the magnetic strange parameter, $\mu_{s}$, is represented by the black band. The range of variation considered for $\mu_{s}$ is consistent with its prediction from the fit to the elastic data. The uncertainty in the isovector contribution of the axial-vector form factor leads to the green band shown in the figure.

\section{Conclusions}

We have presented a brief summary of some relevant results on PV elastic and quasielastic electron scattering published in previous studies $[15,20,25,14,26]$.

\footnotetext{
${ }^{3}$ In the exclusive reaction the ejected nucleon is detected in coincidence with the final electron, $A\left(\vec{e}, e^{\prime} N\right) B$.
} 

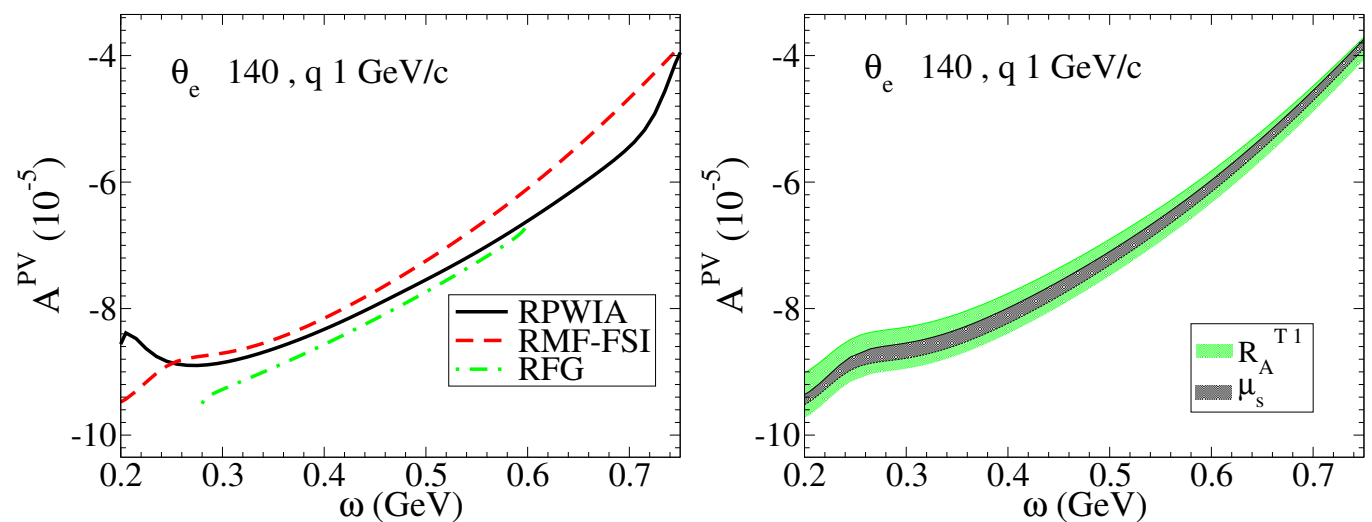

Figure 4: (Left panel) $\mathscr{A}_{Q E}^{P V}$ computed with different models. (Right panel) Effects of magnetic strangeness $\left(\mu_{s}\right)$ and axial isovector radiative corrections $\left(R_{A}^{T=1}\right)$ on $\mathscr{A}_{Q E}^{P V}$ computed with RMF-FSI model. In both panels the PVQE asymmetry is represented as a function of the energy transfer, $\omega$, while the scattering angle and momentum transferred are fixed to $\theta_{e}=140^{\circ}$ and $q=1 \mathrm{GeV} / \mathrm{c}$.

In section 2 we have discussed the relevance of the PV elastic electron scattering asymmetry as an excellent tool to study the WNC form factors. We have shown the results of a statistical analysis ( $\chi^{2}$ fit) of the full set of data using as free parameters the WNC effective vector couplings $\left(\xi_{V}^{p, n}\right)$, the strangeness parameters $\left(\rho_{s}\right.$ and $\left.\mu_{s}\right)$ and the value of the axial-vector form factor at zero $Q^{2}\left(G_{A}^{e p}\right)$. An important result from the fit is the strong correlation existent between $\rho_{s}, \mu_{s}$ and $G_{A}^{e p}$. Also, the fit provides an unexpectedly low value for $G_{A}^{e p}$ that could be understood as a signal of the importance of RC effects in the axial-vector current (significantly higher than the current estimates). This result may also indicate that alternative prescriptions for the $Q^{2}$ dependence of the strange and axial-vector form factors should be explored. Therefore, more studies on RC in the axial sector are essential before definite conclusions can be drawn on the vector strange form factors of the nucleon.

In section 3 we have presented a brief discussion on the PV asymmetry for QE electron-nucleus scattering. Although additional uncertainties arise from the use of a complex nuclear target, we have shown that the PVQE asymmetry can provide nucleonic information that clearly complements the one attached to the PVE case. In particular, measurements of $\mathscr{A}_{Q E}^{P V}$ at backward scattering angles could constrain the RC that enter in the isovector sector of the axial-vector form factor. This analysis, because of the strong correlation between the parameters, is essential in order to provide more accuracy estimates on the electric and magnetic strangeness contributions.

\section{References}

[1] L. Alvarez-Ruso, Y. Hayato and J. Nieves, New J. Phys. 16, 075015 (2014)

[2] E. J. Beise, M. L. Pitt and D. T. Spayde. Prog. Part. Nucl. Phys., 54, 289 (2005).

[3] K. A. Aniol et al. [HAPPEX Collaboration], Phys. Rev. C., 69, 065501 (2004).

[4] K. A. Aniol et al. [HAPPEX Collaboration], Phys. Lett. B., 635, 275 (2006).

[5] K. A. Aniol et al. [HAPPEX Collaboration], Phys. Rev. Lett., 98, 032301 (2007).

[6] Z. Ahmed et al. [HAPPEX Collaboration], Phys. Rev. Lett., 108, 102001 (2012). 
[7] F. E. Maas et al. [PVA4 Collaboration], Phys. Rev. Lett., 93, 022002 (2004).

[8] F. E. Maas et al. [PVA4 Collaboration], Phys. Rev. Lett., 94, 152001 (2005).

[9] S. Baunack et al. [PVA4 Collaboration], Phys. Rev. Lett., 102, 151803 (2009).

[10] D. S. Armstrong et al. [G0 Collaboration], Phys. Rev. Lett., 195, 092001 (2005).

[11] D. Androić et al. [G0 Collaboration], Phys. Rev. Lett., 195, 092001 (2005).

[12] D. Androic et al. [Qweak Collaboration], Phys. Rev. Lett., 111, 141803 (2013).

[13] K. A. Aniol et al. [HAPPEX Collaboration], Phys. Rev. Lett., 96, 022003 (2006).

[14] R. González-Jiménez, J. A. Caballero and T. W. Donnelly, "Parity violation in quasielastic electron nucleus scattering within the relativistic impulse approximation". In preparation (2015).

[15] R. González-Jiménez, J. A. Caballero and T. W. Donnelly, Phys. Rep. 524, 1 (2013).

[16] M. J. Musolf and T. W. Donnelly, J. Dubach, S.J. Pollock, S. Kowalski and E.J. Beise, Phys. Rep. 239, $1 \& 2$ (1994).

[17] A. Airapetian et al., [HERMES Collaboration], Phys. Rev. Lett. 92, 012005 (2004).

[18] V. YU. Alexakhin et al., [COMPASS Collaboration], Phys. Lett. B 647, 8 (2007).

[19] J. Liu, R. D. McKeown and M. J. Ramsey-Musolf, Phys. Rev. C 76, 025202 (2007).

[20] R. González-Jiménez, J. A. Caballero and T. W. Donnelly, Phys. Rev. D. 90, 033002 (2014).

[21] E. L. Lomon, Phys. Rev. C 64, 035204 (2001).

[22] E. L. Lomon, Phys. Rev. C 66, 045501 (2002).

[23] C. Crawford et al., Phys. Rev. C 82, 045211 (2010).

[24] J. Beringer et al., Phys. Rev. D 86, 010001 (2012).

[25] O. Moreno, T. W. Donnelly, R. González-Jiménez and J. A. Caballero, arXiv:1408.3511 [nucl-th] Aug (2014).

[26] R. González-Jiménez, J. A. Caballero and T. W. Donnelly, "Parity violation and dynamical relativistic effects in $\left(\vec{e}, e^{\prime} N\right)$ reactions". In preparation (2015).

[27] T. W. Donnelly, M. J. Musolf, W. M. Alberico, M. B. Barbaro, A. De Pace and A. Molinari, Phys. Rev. A 541, 525 (1992). 